\title{
Topology optimization in magnetic fields using the homogenization design method
}

\author{
Jeonghoon Yoo*,† and Noboru Kikuchi \\ Department of Mechanical Engineering and Applied Mechanics, University of Michigan, Ann Arbor, MI 48109, U.S.A.
}

\begin{abstract}
SUMMARY
To improve the performance of electric machinery, it is necessary to obtain the optimal topology of a structure in magnetic fields. The homogenization design method is applied to obtain the optimal topology. In the method, the change of inner hole size and rotational angle of unit cell determines the optimal material distribution in a design domain and this distribution defines an optimal topology. The objective function is defined as maximizing magnetic mean compliance (MMC). This is the same as maximizing magnetic vector potential and effective to improve the performance of electomagnet. The analysis and optimization is performed based on three-dimensional hexahedral elements. This design method is applied to the H-shaped electromagnet (H-magnet). Copyright (C) 2000 John Wiley \& Sons, Ltd.
\end{abstract}

KEY WORDS: optimal topology; magnetic fields; homogenization design method; hexahedral elements

\section{INTRODUCTION}

The optimal synthesis problem of an electromagnetic system is to obtain the optimal shape which atisfies the prescribed performance subject to some limitations and constraints. A traditional but inefficient way of solving this problem is the trial and error method in which the modification is determined mainly by the designer's intuition and experience. The direct search method is widely used in structural mechanics as well as in electromagnetics. The optimal design to satisfy the objective function can be performed either by the derivative free approach or by the gradientsbased technique. The gradients-based techniques is one of the most powerful methods for the optimization using the finite element method, and it is very popular for the optimization of electromagnetic systems [1-3]. In this method, some control points, which are usually specific nodes in a finite element model, are designated and the positions of the points are changed to satisfy the prescribed performance. For the optimization process, a sensitivity analysis plays a central role. The definition of sensitivity must correspond to the objective function and the design variable. However, this method determines only the outer shape of the design domain for

\footnotetext{
* Correspondence to: Jeonghoon Yoo, Department of Mechanical Engineering and Applied Mechanics, University of Michigan, Ann Arbor, MI 48109, U.S.A.

† E-mail: yoojh@engin.umich.edu
} 
the optimization. Therefore, it can be classified as a shape optimization rather than a topology optimization.

Since it was first introduced to electromagnetic field analysis by Silvester [4], the finite element method has been preferred, especially for the analysis of a field which has complex boundary shapes. Schmit [5] first coupled the finite element method with non-linear mathematical programming for optimal structural design. In electromagnetic fields, Marrocco and Pironneau's work [6] can be regarded as a pioneer work which developed the optimal design of a magnet combined with Lagrangian finite elements. Nakata and Takahashi [7] presented a new design method using the finite element method and the gradient method. Using the finite element method, the performance of an electromagnetic system can be calculated with reasonable accuracy.

Since firstly introduced for topology optimization by Bendsøe and Kikuchi [8], topology optimization using the homogenization design method has been successfully applied to elastic structure cases $[9,10]$. This method is based on the homogenization theory and the finite element method. As many other optimization processes, this method is composed of two modules, which are the finite element analysis module and the optimization module.

In this paper, topology optimization using the homogenization design method is extended to obtain the optimal topology of a structure in magnetic fields. In the iteration process for the optimization in magnetic fields, the homogenized magnetic permeability value is decided corresponding to design variables which are inner hole size and rotational angle of each unit cell. Using the permeability value, the objective function based on magnetic energy is computed. The optimal material distribution is decided during the optimization process and this determines the optimal topology. Sequential linear programming is used for the optimization process since large number of design variables and the negative sensitivity value must be managed.

\section{HOMOGENIZATION IN MAGNETIC FIELDS}

In topology optimization using the homogenization design method, the optimal shape of a structure is determined by the optimal material distribution. The design domain, $\Omega$, is composed of a composite material with perforated microstructures as shown in Figure 1. The design domain is expressed as finite elements. It is assumed that each element is composed of infinite number of unit cells. The design domain has given boundary conditions $\partial \Omega_{d}$ and $\partial \Omega_{J}$, which are the potential boundary condition and current density boundary condition, respectively. The unit cell of the microstructure has a rectangular hole, as shown in Figure 1. If the hole size of the unit cell is 0 , the unit cell becomes a solid. On the other hand, if the hole size is the same as the unit cell size, the unit cell becomes a void. During the optimization process, the material is transferred from one part to another part in the design domain and finally the optimal distribution of the material is determined.

The optimization problem using the homogenization method is defined by the total potential energy/mean compliance of a design domain. In magnetic fields, the weak form of magnetic energy can be formulated using homogenized magnetic permeability. Thus, to obtain the homogenized properties of a given microstructure, the unit cell problem must be solved. The microstructure model in a three-dimensional case is shown in Figure 2. The unit cell of three-dimensional microstructure has a body hole of width $1-a$, depth $1-b$, and height $1-c$. The size of body hole $\mathbf{D}=\{a, b, c\}$, and the rotation angle $\Theta=\{\varphi, \theta, \psi\}$ are the design variables. 


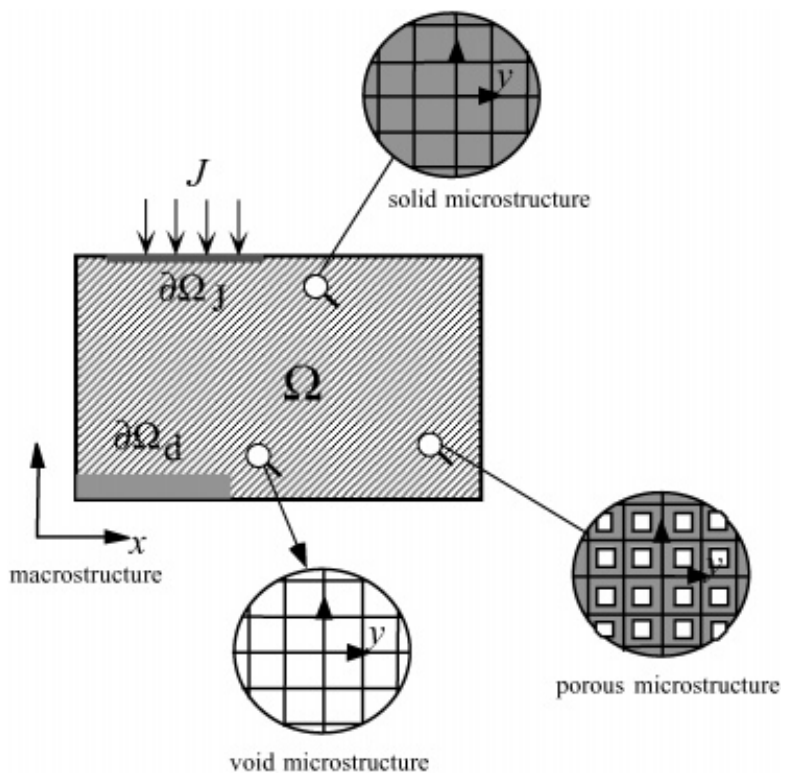

Figure 1. Macro-design domain and porous microstructures.

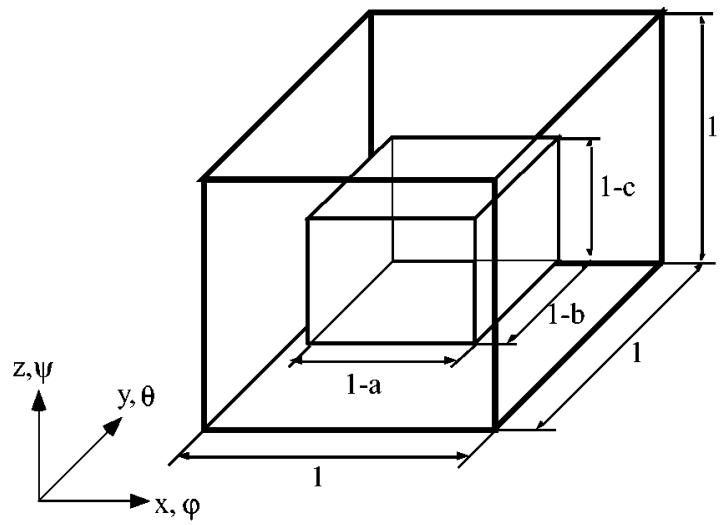

Figure 2. Unit cell for a three-dimensional microstructure.

These design variables are changed during the optimization process and define the material density of each element. Assuming $\mathbf{R}(\Theta)$ to be the rotational matrix based on rotational and $\Theta$, magnetic permeability is finally computed using the following equation

$$
\mu^{\mathrm{h}}=\mathbf{R}(\Theta)^{\mathrm{T}} \mu^{\mathrm{H}}(\mathbf{D}) \mathbf{R}(\Theta)
$$


where $\boldsymbol{\mu}^{\mathrm{H}}(\mathbf{D})$ represents homogenized magnetic permeability without considering rotation and $\mu^{\mathrm{h}}$ represents the final value of homogenized magnetic permeability.

\section{Mathematical formulation}

The homogenization method can be used for periodic materials which are composed of a periodic repetition of a unit cell. Then, in the magnetic field analysis, the constitutive parameters, such as homogenized permeability, can be obtained using the formulation developed by SanchezPalencia [11] and Bytner et al. [12].

The Maxwell equation for magnetic fields is written as

$$
\begin{gathered}
\nabla \cdot \mathbf{B}=\mathbf{0} \\
\nabla \times \mathbf{H}=\mathbf{J}+\frac{\partial \mathbf{D}}{\partial t}
\end{gathered}
$$

where $\mathbf{B}$ is the magnetic flux density and $\mathbf{H}$ is the magnetic field strength. $\mathbf{J}$ and $\mathbf{D}$ represents the current density and the electric flux density, respectively. The constitutive relation between $\mathbf{H}$ and $\mathbf{B}$ is written as

$$
\mathbf{B}=\boldsymbol{\mu} \mathbf{H}
$$

In magnetic fields, $\boldsymbol{\mu}$ can be assumed as an orthotropic matrix since shear term of the matrix has negligible value and is usually not considered for flux density calculation. Therefore, it can be expressed such that

$$
\boldsymbol{\mu}=\left[\begin{array}{ccc}
\mu_{x} & 0 & 0 \\
0 & \mu_{y} & 0 \\
0 & 0 & \mu_{z}
\end{array}\right]
$$

Let the permeability, $\boldsymbol{\mu}$, be Y-periodic functions of $\mathbf{y}=\mathbf{x} / \mathbf{\varepsilon}$ satisfying the following condition

$$
\boldsymbol{\mu}^{\varepsilon}(\mathbf{x}, \mathbf{y})=\boldsymbol{\mu}(\mathbf{x}, \mathbf{y}+\mathbf{Y})
$$

Here, $\mathbf{x}$ is the co-ordinate of a point in macro-scale and it has three directions, $x_{1}, x_{2}$, and, $x_{3}$ for three-dimensional cases. On the contrary, $\mathbf{y}$ is the co-ordinate defined in a unit cell characterizing a microstructure and it also has three directions, $y_{1}, y_{2}$, and, $y_{3}$ for three-dimensional cases. $\boldsymbol{\varepsilon}$ represents the size of the microstructure and $\mathbf{Y}$ is the size of a unit cell. Let coordinate $\mathbf{x}_{0}$ be the origin of the unit structure for the microstructure. The relation between these variables is written as

$$
\mathbf{y}=\frac{\mathbf{x}-\mathbf{x}_{0}}{\varepsilon}
$$


Using this relation to expand $\mathbf{H}$, it becomes

$$
\mathbf{H}^{\varepsilon}=\mathbf{H}_{0}(\mathbf{x}, \mathbf{y})+\boldsymbol{\varepsilon} \mathbf{H}_{1}(\mathbf{x}, \mathbf{y})
$$

If only the magnetic field of the macro-scale is considered, Equation (8) simply becomes $\mathbf{H}^{\varepsilon}=\mathbf{H}_{0}(\mathbf{x})$. However, since the field strength of the micro-scale is considered, the second term of Equation (8) must be added. Using Equations (7) and (8) into Equation (3), a hierarchy of equations can be obtained. Terms for $\varepsilon^{-1}$ and $\varepsilon^{0}$ of Equation (3) are derived as

$$
\begin{gathered}
\nabla_{y} \times \mathbf{H}_{0}=0 \\
\nabla_{x} \times \mathbf{H}_{0}+\nabla_{y} \times \mathbf{H}_{1}=J_{0}+\frac{\partial \mathbf{D}_{0}}{\partial t}
\end{gathered}
$$

And the term for $\varepsilon^{-1}$ of Equation (2) is derived as

$$
\operatorname{div}_{y}\left(\boldsymbol{\mu} \mathbf{H}_{0}\right)=0
$$

Equation (9) implies that $\mathbf{H}_{0}$ are gradients in $\mathbf{y}$. Thus, it can be rewritten as

$$
\mathbf{H}_{0}=\nabla_{y} \Psi+\int_{Y} \mathbf{H}_{0} \mathrm{~d} \mathbf{y}
$$

where $\Psi$ is a $Y$-periodic function and has a scalar value. $\mathbf{H}_{0}$ is also function of $\mathbf{y}$. Therefore, not only the macro-scale value but also the micro-scale value must be considered in $\mathbf{H}_{0}$. In Equation (12), the first term on the right-hand side expresses the micro-scale value. Substituting Equation (12) into Equation (11), the following equation can be derived:

$$
\operatorname{div}_{y}\left(\boldsymbol{\mu} \nabla_{y} \Psi\right)+\operatorname{div}_{y}\left(\boldsymbol{\mu} \int_{Y} \mathbf{H}_{0} \mathrm{~d} \mathbf{y}\right)=\operatorname{div}_{y}\left(\boldsymbol{\mu} \nabla_{y} \Psi+\boldsymbol{\mu} \tilde{\mathbf{H}}_{0}\right)=0
$$

where $\tilde{\mathbf{H}}_{0}$ is only a function of $\mathbf{x}$. Considering the relation of Equation (13), $\Psi$ can be written as

$$
\Psi=\chi(\mathbf{y}) \int_{Y} \mathbf{H}_{0} \mathrm{~d} \mathbf{y}=\chi(\mathbf{y}) \tilde{\mathbf{H}}_{0}
$$

where $\chi(\mathbf{y})$ is the characteristic magnetic field strength in the unit cell. The characteristic magnetic field strength $\chi(\mathbf{y})$ can be determined by solving the following equation which is obtained by Equations (13) and (14):

$$
\operatorname{div}_{y}\left[\boldsymbol{\mu}(\mathbf{y}) \nabla_{y} \chi(\mathbf{y}) \tilde{\mathbf{H}}_{0}+\boldsymbol{\mu}(\mathbf{y}) \tilde{\mathbf{H}}_{0}\right]=0
$$


Since $\tilde{\mathbf{H}}_{0}$ is a function of only $\mathbf{x}$, Equation (15) can be expressed as

$$
\operatorname{div}_{y}\left[\boldsymbol{\mu}(\mathbf{y}) \nabla_{y} \chi(\mathbf{y})+\boldsymbol{\mu}(\mathbf{y})\right]=\mathbf{0}
$$

Using variational calculus and integration by parts, this equation can be written as a tensor form

$$
\int_{Y} \mu_{i j} \frac{\partial \chi_{i}}{\partial y_{i}} \frac{\partial v_{p}}{\partial y_{i}} \mathrm{~d} y=-\int_{Y} \mu_{i j} \frac{\partial v_{p}}{\partial y_{i}} \mathrm{~d} y
$$

where $v_{p}$ is defined in space $V$ which is expressed as

$$
V=\left\{v ; v_{p} \in H^{1}(\Omega),\left.v_{p}\right|_{\partial_{1} \Omega}=0\right\}
$$

where $H^{1}$ is the Sobolev space differentiable at least once. By solving Equation (17) the characteristic magnetic field strength $\chi(\mathbf{y})$ can be obtained. The constitutive relation defined in Equation (4) can be expressed by mean values such that

$$
\int_{Y} \mathbf{B}_{0} \mathrm{~d} y=\boldsymbol{\mu}^{\mathrm{h}} \int_{Y} \mathbf{H}_{0} \mathrm{~d} \mathbf{y}
$$

where the homogenized permeability $\boldsymbol{\mu}^{\mathrm{h}}$ is defined using Equation (15) and the characteristic magnetic field strength is defined as follows:

$$
\boldsymbol{\mu}^{\mathrm{h}}=\int_{Y} \boldsymbol{\mu} \mathrm{d} \mathbf{y}+\int_{Y} \boldsymbol{\mu} \nabla_{y} \chi(\mathbf{y}) \mathrm{d} \mathbf{y}
$$

This $\boldsymbol{\mu}^{\mathrm{h}}$ matrix is determined during the optimization process associated with the change of design variables.

\section{OPTIMIZATION PROBLEM}

In this section, the optimization problems are defined to obtain the optimal shapes which maximize MMC in a design domain for linear cases. Since the homogenization method is used as a design methodology, the objective function is expressed as an energy formulation. The computation of the sensitivity is also important to obtain new design variables. For the optimization solver, sequential linear programing is used.

\section{Magnetic energy}

The structural optimization problem is based on the energy value of a structure. The objective of the optimization is generally formulated as maximizing or minimizing the energy. Thus, for a structural optimization in magnetic fields, the magnetic energy must be calculated. In a region 
$V$, the magnetic energy can be defined such that $[13,14]$

$$
W_{\mathrm{m}}=\frac{1}{2} \int_{V} \mathbf{B} \cdot \mathbf{H} \mathrm{d} v=\frac{1}{2} \int_{V} \mathbf{B}^{\mathrm{T}} \frac{1}{\boldsymbol{\mu}^{\mathrm{h}}} \mathbf{B} \mathrm{d} v
$$

where $\boldsymbol{\mu}^{\mathrm{h}}$ is the homogenized magnetic permeability. B can be calculated using the vector potential, A. Thus, Equation (21) can be rewritten as

$$
W_{\mathrm{m}}=\frac{1}{2} \int_{V}(\nabla \times \mathbf{A}) \cdot \mathbf{H} \mathrm{d} v
$$

Using a vector identity and using $\nabla \times \mathbf{H}=\mathbf{J}$, we obtain

$$
W_{\mathrm{m}}=\frac{1}{2} \int_{V} \mathbf{J} \cdot \mathbf{A} \mathrm{d} v+\frac{1}{2} \int_{V} \nabla \cdot(\mathbf{A} \times \mathbf{H}) \mathrm{d} v
$$

Applying Stoke's theorem, Equation (23) can be rewritten as

$$
W_{\mathrm{m}}=\frac{1}{2} \int_{V} \mathbf{J} \cdot \mathbf{A} \mathrm{d} v+\frac{1}{2} \oint_{S} \mathbf{A} \times \mathbf{H} \cdot \mathrm{d} S
$$

Let the surface over which the second integral performs go to infinity. Then since $\mathbf{A} \propto 1 / r$, $\mathbf{H} \propto 1 / r^{2}$, and $S \propto r^{2}$, the second term becomes zero [14]. Therefore, the magnetic energy is defined as

$$
W_{\mathrm{m}}=\frac{1}{2} \int_{V} \mathbf{B}^{\mathrm{T}} \frac{1}{\mu^{\mathrm{h}}} \mathbf{B} \mathrm{d} v=\frac{1}{2} \int_{V} \mathbf{J} \cdot \mathbf{A} \mathrm{d} v
$$

The optimization process is performed using the finite element method. Thus, the magnetic energy is calculated based on each of the finite elements which compose the design domain. The magnetic energy of each element in a design domain can be calculated using the following equation:

$$
U_{\mathrm{m} e}=\frac{1}{2} \int_{\Omega_{e}} \mathbf{B}_{e}^{\mathrm{T}} \mathbf{M}_{e}^{\mathrm{h}} \mathbf{B}_{e} \mathrm{~d} \Omega
$$

where $\mathbf{B}_{e}$ is the flux density of the element and $\mathbf{M}_{e}^{\mathrm{h}}$ is the homogenized permeability matrix. $\mathbf{M}_{e}^{\mathrm{h}}$ is defined as follows:

$$
\mathbf{M}_{e}^{\mathrm{h}}=\left[\begin{array}{ccc}
\frac{1}{\mu_{x}^{\mathrm{h}}} & 0 & 0 \\
0 & \frac{1}{\mu_{y}^{\mathrm{h}}} & 0 \\
0 & 0 & \frac{1}{\mu_{z}^{\mathrm{h}}}
\end{array}\right]
$$


where $\mathbf{M}_{e}^{\mathrm{h}}$ is assumed to be orthotropic. The value of $\mathbf{B}_{e}$ is calculated from vector potential $\mathbf{A}_{e}$ using the following relation

$$
\mathbf{B}_{e}=\nabla \times \mathbf{A}_{e}
$$

Therefore, the total magnetic energy of a design domain can be calculated by adding the magnetic energy of each element composing the design domain.

\section{Optimization problem for a linear case}

The objective of the optimal design in magnetic fields can be defined as maximizing MMC in a design domain. If this objective is applied, the volume of the optimal structure decreases as MMC increases. Thus, a minimum bound of total volume must be constrained. In electromagnetics, the total potential energy can be defined as

$$
F_{\mathrm{m}}=\frac{1}{2} \int_{V} \mathbf{B}^{\mathrm{T}} \mathbf{M B} \mathrm{d} v-\int_{V} \mathbf{J} \cdot \mathbf{A} \mathrm{d} v
$$

Considering the relation shown in Equation (25), minimizing the total potential energy is the same as maximizing MMC

$$
\mathrm{MMC}=\int_{V} \mathbf{J} \cdot \mathbf{A} \mathrm{d} v
$$

Considering the objective and the volume constraint, the optimization problem can be defined as follows:

$$
\begin{array}{ll}
\underset{x}{\operatorname{maximize}} & \mathrm{MMC}=\int_{V} \mathbf{J} \cdot \mathbf{A} \mathrm{d} v \\
\text { subject to } & \sum_{e=1}^{N} v_{e}-V \geqslant 0
\end{array}
$$

where $\mathbf{X}$ is the design variable. The objective function is the same as maximizing vector potential A since $\mathbf{J}$ is usually fixed. However, this cannot guarantee maximizing $\mathbf{B}$ because $\mathbf{B}$ is defined as curl of $\mathbf{A}$ as in Equation (28). During the iteration for maximizing $\mathrm{MMC}$, the volume decreases. Thus, the lower bound of total volume must be constrained as in Equation (31).

The flux linkage can be found as

$$
\varphi=\frac{\int_{V} \mathbf{J} \cdot \mathbf{A} \mathrm{d} v}{I}
$$


Using the definition of magnetic flux, $\phi=\varphi / N$, the mean compliance can be rewritten as

$$
\mathrm{MC}=\frac{I \varphi}{N} N=N I \phi
$$

where number of turns $N$ and current $I$ are already decided. Thus, the objective is the same as maximizing magnetic flux.

Letting $X$ be the design variable and taking a variation of $F_{\mathrm{m}}$, the derivative of potential energy becomes

$$
\begin{aligned}
\delta F_{\mathrm{m}}= & \int_{V} \mathbf{B}(\mathbf{A})^{\mathrm{T}} \mathbf{M B}\left(\delta \mathbf{A}+\frac{\partial \mathbf{A}}{\partial X} \delta X\right) \mathrm{d} \Omega+\frac{1}{2} \int_{V} \mathbf{B}(\mathbf{A})^{\mathrm{T}} \frac{\partial \mathbf{M}}{\partial X} \mathbf{B}(\overline{\mathbf{A}}) \mathrm{d} \Omega \delta X \\
& -\int_{V} \mathbf{J} \cdot\left(\delta \mathbf{A}+\frac{\partial \mathbf{A}}{\partial X} \delta X\right) \mathrm{d} v
\end{aligned}
$$

The first and third terms can be cancelled out. Thus, the sensitivity of $F_{\mathrm{m}}$ for design variable $X$ can be defined as

$$
\frac{\partial F_{\mathrm{m}}}{\partial X}=\frac{1}{2} \int_{V} \mathbf{B}^{\mathrm{T}} \frac{\partial \mathbf{M}}{\partial X} \mathbf{B} \mathrm{d} \Omega
$$

\section{NUMERICAL EXAMPLES}

In order to verify the optimization method explained previous sections, two examples are provided. The first example is about the maximizing MMC of an $\mathrm{H}$-magnet. The second one is similar to the first example. The design objective is to improve the performance by maximizing magnetic vector potential. However, three layer finite element model is used to define a three dimensional structure.

Example 1: Maximizing mean compliance in a linear case I

The optimization problem defined in Equation (31) is applied to obtain the optimal shape of an $\mathrm{H}$-magnet. Figure 3(a) shows the cross-section of an H-magnet. $\Omega_{\mathrm{f}}$ represents the iron part and $\Omega_{\mathrm{c}}$ represents the copper wire where the current density of $5 \times 10^{6} \mathrm{~J} / \mathrm{m}^{2}$ is applied. $\Omega_{\mathrm{a}}$ represents an empty portion of the magnet. The initial relative permeability of the iron is assumed as 4000 . The object of optimal design is to maximize MMC at the end of subregion $\Omega_{\mathrm{f}}$ which is adjacent to the empty portion. For the finite element model, only one quarter of the $\mathrm{H}$-magnet can be considered as shown in Figure 3(b) because of the symmetry. The Dirichlet boundary condition is applied along the $\Gamma_{0}$ region and the Neumann boundary condition is applied along the $\Gamma_{1}$ region. Figure 4 shows the initial finite element model which is one layer deep in the $z$-direction using hexahedral eight-node elements. 


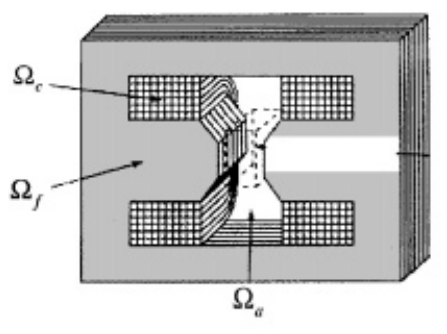

(a)

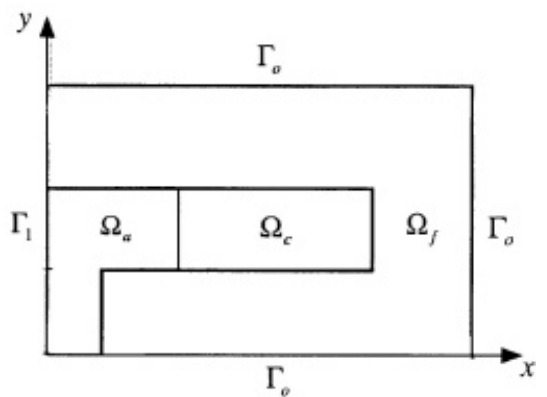

(b)

Figure 3. Shape of an H-magnet: (a) cross-sectional view; (b) quarter model for analysis.

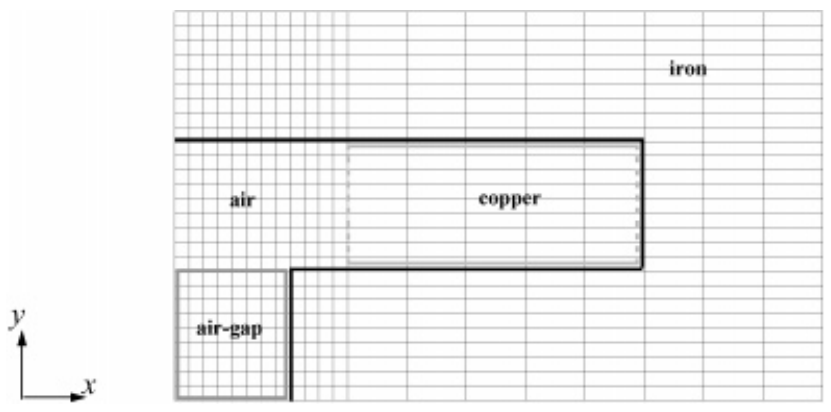

Figure 4. An initial finite element model of an quarter of an H-magnet.

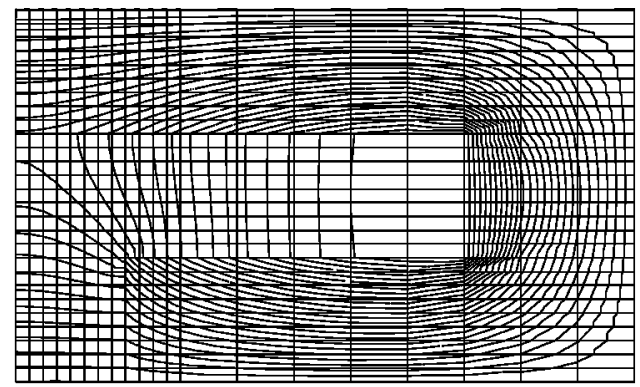

(a)

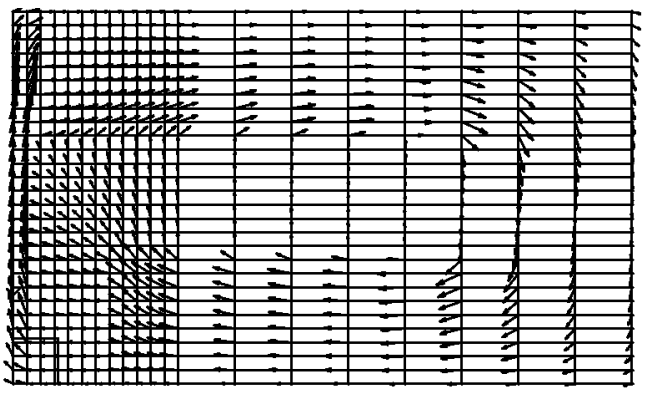

(b)

Figure 5. Finite element analysis results of an H-magnet: (a) vector potential; (b) flux density.

Figure 5(a) shows a contour plot of the vector potential and Figure 5(b) shows a vector plot of the magnetic flux density for the finite element model shown in Figure 4. Both analyses were performed assuming that the value of the magnetic permeability was linear. As can be seen from the vector plot of the flux density, in the air gap of the H-magnet the vectors are aligned in the 


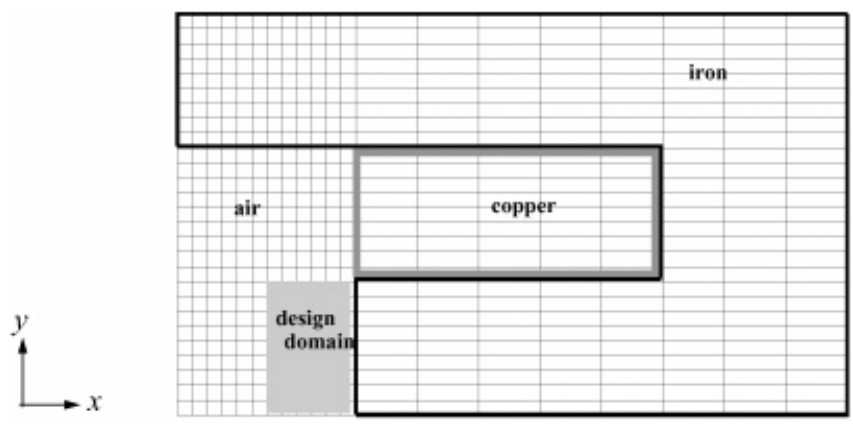

Figure 6. A design domain for the optimization.

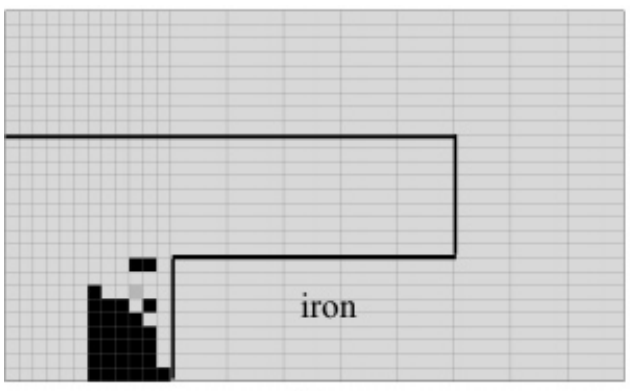

(a)

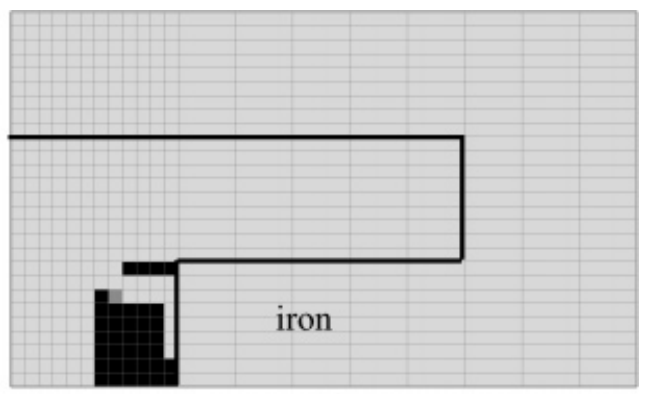

(b)

Figure 7. Optimal shapes of the design domain for different volume constraints: (a) 60 per cent volume constraint; (b) 70 per cent volume constraint.

negative $x$-direction. Also, because of the effect of the iron in the upper part, the vector plots in the empty portion $\left(\Omega_{\mathrm{a}}\right.$ of Figure $\left.3(\mathrm{~b})\right)$ are directed in the positive $y$-direction.

The design domain is defined in the finite element model as shown in Figure 6. The material density of other parts are fixed during optimization. The model is composed of hexahedral eight-node elements one layer deep in the $z$-direction. Only the shaded area of the iron is assigned as a design domain because the air gap is necessary for the original function of the H-magnet. Therefore, the optimal design is a topology optimization of the shaded area, and the design domain is composed of 54 elements of the total 540 elements.

The objective function which is to maximize MMC is applied to the design domain of Figure 6. Figure 7(a) shows the optimal shape for a 60 per cent volume constraint and Figure 7(b) shows the optimal shape for a 70 per cent volume constraint. The black parts represent high material density. As can be seen from these figures, the optimal shapes are similar. However, Figure 7(b) shows a better result since no checkerboard pattern appears and the optimal shape is well connected to the original iron part without an air gap. Figure 8 shows the convergency history of the optimization process for a 70 per cent volume constraint. The objective in the graph is 


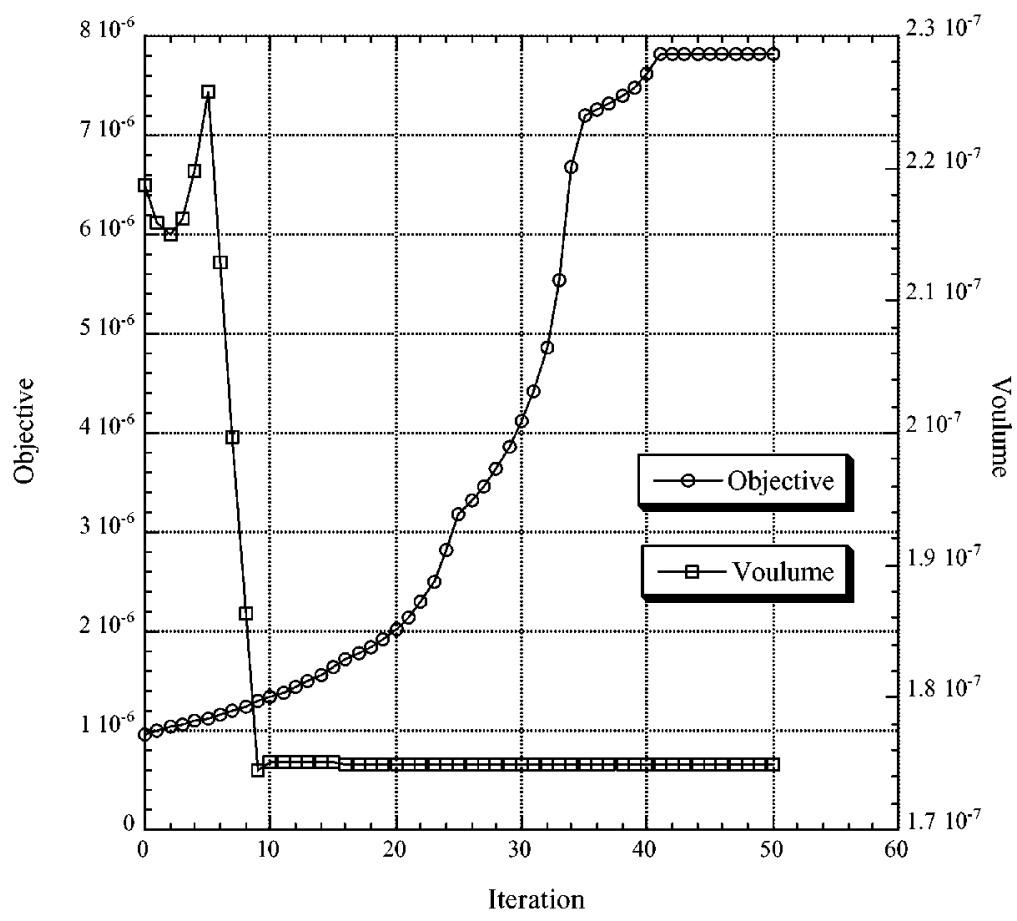

Figure 8. Convergency history of optimization for maximizing mean compliance of the H-magnet (70 per cent volume constraint).

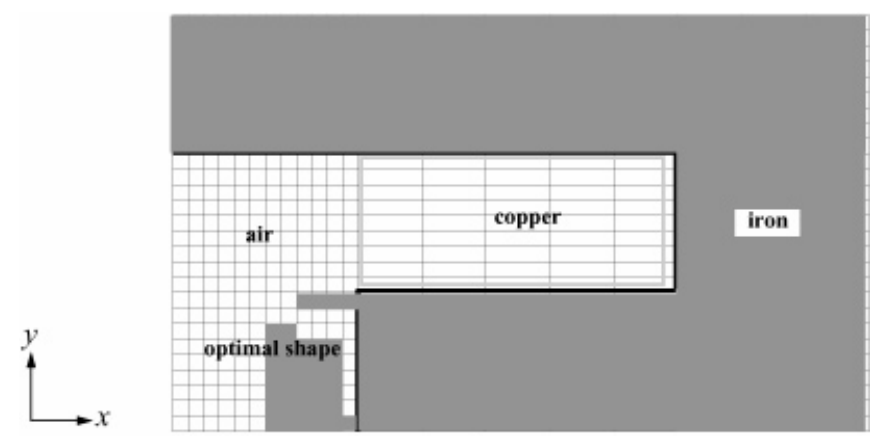

Figure 9. An optimal shape of the H-magnet.

MMC. As can be seen from the figure, the value of the objective function converges well after 40 iterations.

Based on the optimal shape, as displayed in Figure 7(b), the optimal design for the H-magnet is suggested. The shape of the end part of the iron is changed as shown in Figure 9. There is 
Table I. Comparison between the original model and the optimized model in the design domain.

\begin{tabular}{lccc}
\hline & Initial model & Optimized model & Increase (\%) \\
\hline Averaged vector potential $\mathbf{A}_{z}$ & $-0.14466 \mathrm{E}-02$ & $-0.26463 \mathrm{E}-02$ & 82.936 \\
Averaged flux density $\mathbf{B}_{x}$ & $-0.11623 \mathrm{E}+00$ & $-0.13646 \mathrm{E}+00$ & 17.399 \\
Averged flux density $\mathbf{B}_{y}$ & $0.42568 \mathrm{E}-01$ & $0.82186 \mathrm{E}-01$ & 93.072 \\
Total magnetic energy $(\mathbf{J})$ & $0.2989 \mathrm{E}-05$ & $0.6904 \mathrm{E}-05$ & 130.98 \\
\hline
\end{tabular}

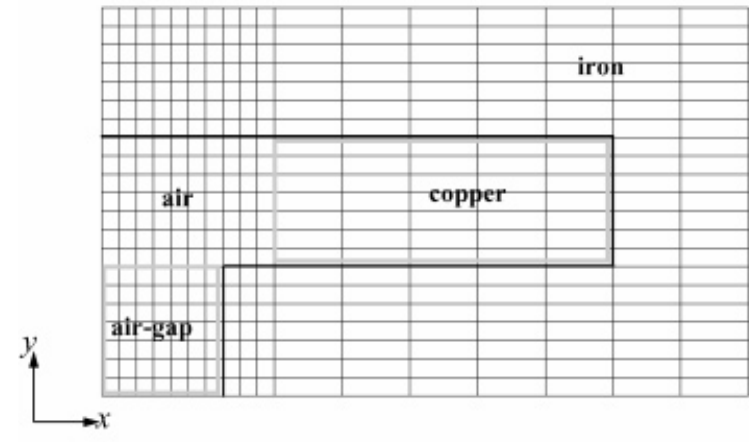

(a)

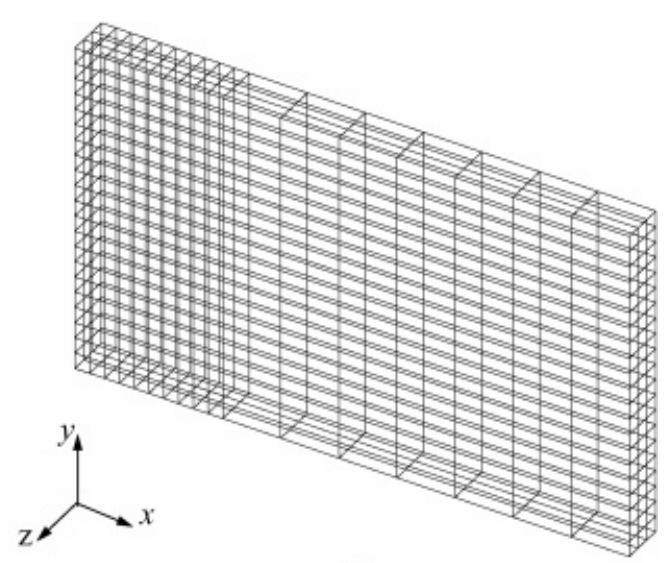

(b)

Figure 10. An initial finite element model of an quarter of an H-magnet: (a) two-dimensional view; (b) three-dimensional view.

a $\neg$-shaped void in the optimized model based on the result shown in Figure 7(b). Using this model, finite element analysis is performed. In magnetic fields, the vector potential value increases by maximizing MMC since the current density value is fixed. This tendency is similar to the case in which the homogenization design method is applied to a structural design for maximizing displacement with fixed external forces. In elastic structure analyses, the increase of strain can be expected if the displacement value is increased. However, in magnetic fields, we cannot guarantee the increase of flux density value although the vector potential value increases. This is caused by the fact that the flux density if defined by the curl of the vector potential while the strain is defined by the divergence of the displacement.

Table I shows the comparison between the original model and the optimized model. As mentioned above, we can see the large increase of the averaged value of vector potential and the total magnetic energy in the design domain. This result can be estimated because the optimization process is maximizing MMC defined by Equation (30) while the current density value is fixed. However, we can see that the flux density values are also increased. This is mainly caused by the fact that the current density is applied only in $z$-direction in this example. The reason why the increase rate of averaged $B_{x}$ is smaller than the increase rate of averaged $B_{y}$ can be estimated that the existence of the upper iron part affects the flux flow in the design domain. 


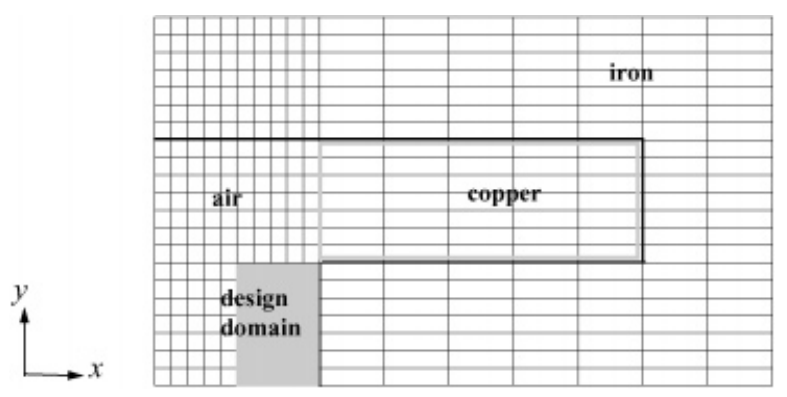

Figure 11. A design domain for the optimization.

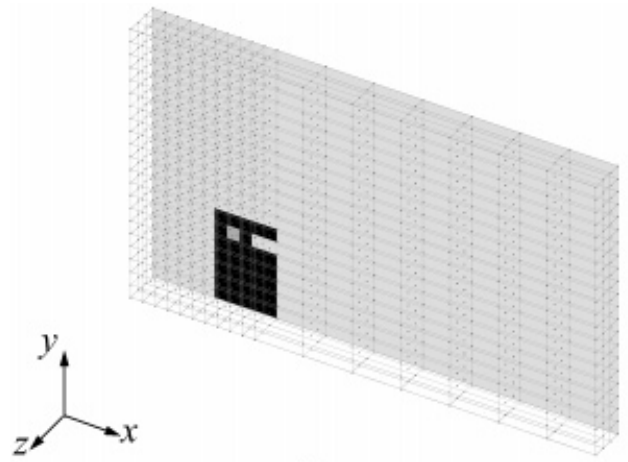

(a)

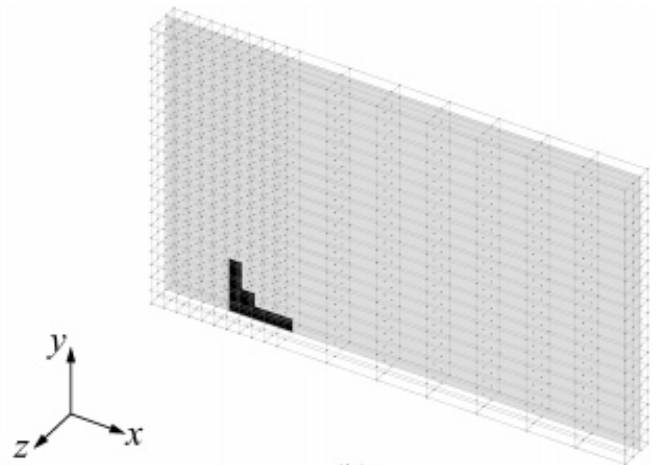

(b)

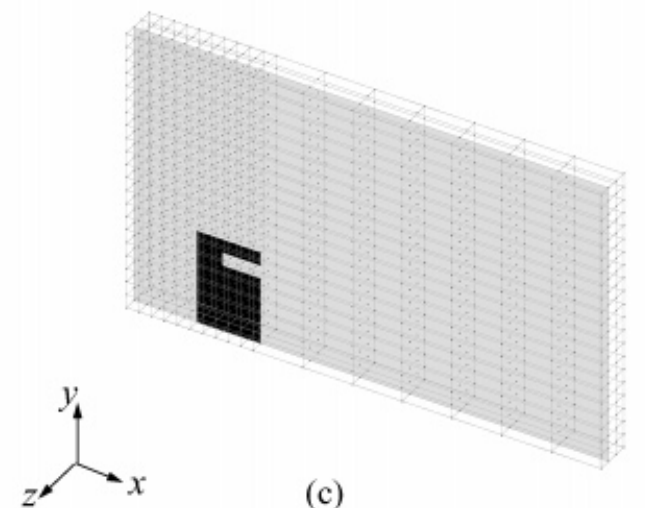

Figure 12. Optimal shape of the design domain at different cross-sections: (a) $\mathrm{z}=0.0$; (b) $\mathrm{z}=0.02$; (c) $\mathrm{z}=0.04$.

Example 2: Maximizing mean compliance in a linear case II

In this example, an H-magnet is used to maximize MMC as in Example 1. However, in this case, a three-layer finite element model in the $z$-direction is used while only one-layer finite element model was used in Example 1. Figure 10(a) shows the finite element model of the H-magnet. The 


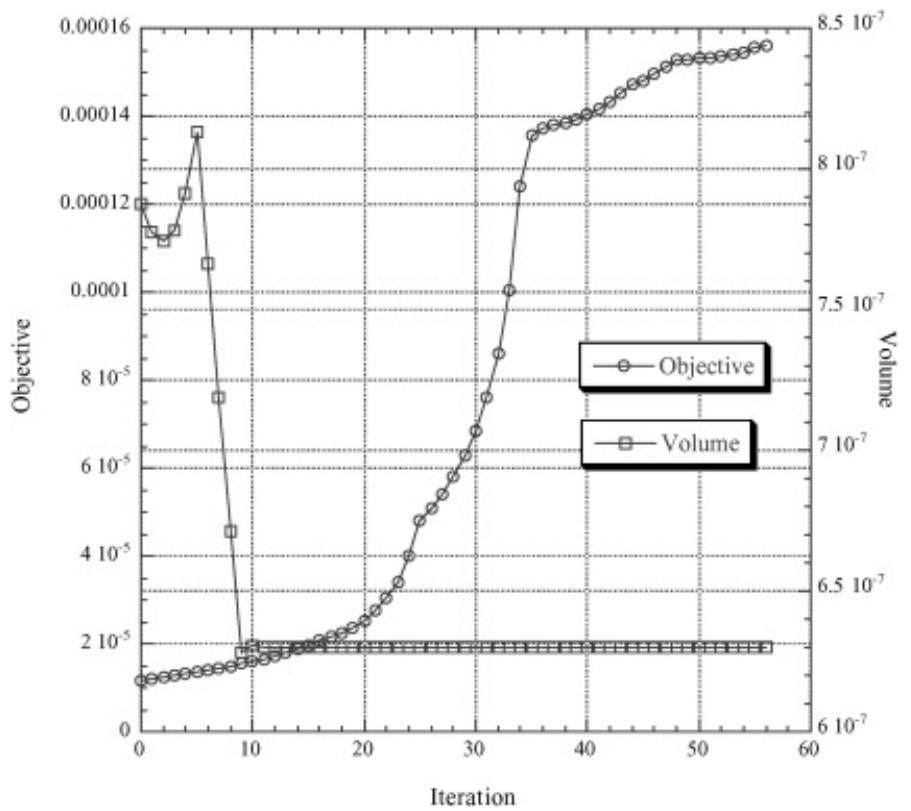

Figure 13. Convergency history of optimization for maximizing mean compliance of the H-magnet (three layer model, 70 per cent volume constraint).
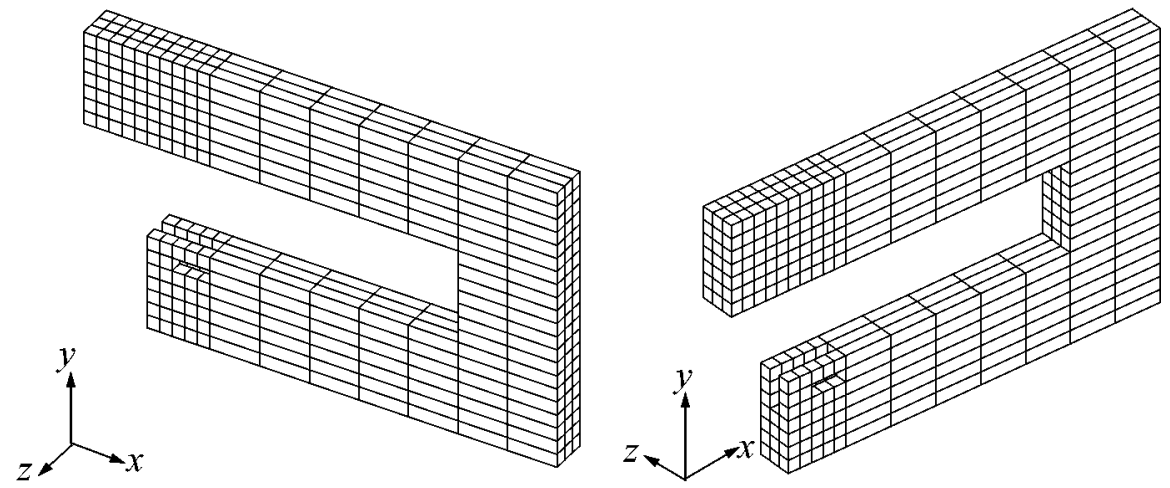

Figure 14. An optimized model of the H-magnet.

original shape of the H-magnet is similar to the original model used in Example 1. Figure 10(b) shows the model in three-dimensional view and it shows the three-layer finite element model in the $z$-direction.

As in Example 1, the objective function, which is maximizing the mean compliance in a design domain, is used for the optimization process. Figure 11 shows the design domain which is located at the end part of the center core. The volume constraint is fixed as 70 per cent to compare the 
Table II. Comparison between the original model and the optimized model in the design domain.

\begin{tabular}{lccr}
\hline & Initial model & Optimized model & Increase (\%) \\
\hline Averaged vector potential $\mathbf{A}_{z}$ & $-0.16302 \mathrm{E}-02$ & $-0.35650 \mathrm{E}-02$ & 118.629 \\
Averaged flux density $\mathbf{B}_{x}$ & $-0.17673 \mathrm{E}+00$ & $-0.18269 \mathrm{E}+00$ & 3.367 \\
Averged flux density $\mathbf{B}_{y}$ & $0.63356 \mathrm{E}-01$ & $0.16714 \mathrm{E}+00$ & 163.811 \\
Total magnetic energy $(\mathbf{J})$ & $0.2793 \mathrm{E}-04$ & $0.9706 \mathrm{E}-04$ & 226.472 \\
\hline
\end{tabular}

optimal shape with the optimal shape obtained in Example 1. Figure 12 shows the optimal shapes of the design domain at three different cross sections which are at $z=0.0, z=0.02$, and $z=0.04$. As in Figure 7, black parts represents high material density. The optimal shapes at first and third layers are similar to each other because of the symmetry. However, Figure 12(b) shows that only a few number of elements are assigned as solid elements in the middle section. Figure 13 shows the convergence history for the optimization process. It can be confirmed that the objective function converges well after 50 iterations. Based on these results, an optimized model can be suggested as shown in Figure 14.

Table II shows the comparison of the averaged vector potential, the averaged flux density, and the magnetic energy in the design domain between the original model and the optimized model. As similar as in Example 1, we can see the large increase of the averaged vector potential value and the total magnetic energy in the design domain. However, comparing to the result of Example 1, the increase of flux density, especially the increase of the averaged $B_{x}$ is small although the increase of the averaged $B_{y}$ is very large. From these results, it can be estimated that the effect of the upper iron part is very large for the three-layer finite element model since the optimal shape has a large void part in the middle layer of the design domain as shown in Figure 12(b).

\section{CONCLUSION}

The homogenization design method is applied to obtain the optimal shape of a structure in magnetic fields. The homogenized permeability values were obtained applying the homogenization method to a microstructure and these values were used as important input data in the optimization process.

The optimization problems were defined to maximize MMC for linear cases. From the analysis results, it was verified that the newly developed optimization process is useful to increase the averaged vector potential and the total magnetic energy in a design domain.

\section{REFERENCES}

1. Salon SJ, Istfan B. Inverse nonlinear finite element problems. IEEE Transactions on Magnetics 1986; 22(5):817-818.

2. Istfan B, Salon SJ. Inverse nonlinear finite element problems with local and global constraints. IEEE Transactions on Magnetics 1988; 24(6):2568-2572.

3. Gitosusatro S, Coulomb JL, Sabonnadiere JC. Performance derivative calculations and optimization process. IEEE Transactions on Magnetics 1989; 25(4):2834-2839.

4. Silvester PP. Finite element solution of homogeneous waveguide problems. Alta Frequenza 1969; 38:313-317.

5. Schmit LA. Structural design by systematic synthesis. Proceedings of 2nd ASCE Conference on Electronic Computation, Pittsburgh, PA, 1960; 105-122. 
6. Marrocco A, Pironneau O. Optimum design with Lagrangian finite elements; design of an electromagnet. Computer Methods in Applied Mechanics and Engineering 1978; 15:277-308.

7. Nakata T, Takahashi N. New design method of permanent magnets by using the finite element method. IEEE Transactions on Magnetics 1983; 19(6):2494-2497.

8. Bendsøe MP, Kikuchi N. Generating optimal topologies in structural design using a homogenization method. Computer Methods in Applied Mechanics and Engineering 1988; 71:197-224.

9. Suzuki K, Kikuchi N. A homogenization method for shape and topology optimization. Computer Methods in Applied Mechanics and Engineering 1991; 93: 291-318.

10. Díaz A, Kikuchi N. Solution to shape and topology eigenvalue optimization problems using a homogenization method. International Journal for Numerical Methods in Engineering 1992; 35:1487-1502.

11. Sanchez-Palencia E. Non-Homogeneous Media and Vibration Theory. Springer: Berlin, 1980.

12. Bytner S, Gambin B. Homogenization of heterogeneous elastic conductor in a magnetic field. Journal of Technical Physics 1992; 33(2):219-223.

13. Tärnhuvud T, Reichert K. Accuracy problems and torque calculation in FE-systems. IEEE Transactions on Magnetics 1988; 24(1):443-446.

14. Salon SJ. Finite Element Analysis of Electrical Machines. Kluwer Academic Publishers: Dordrecht, 1995. 